\begin{tabular}{|c|c|c|}
\hline \multirow{3}{*}{$\begin{array}{r}\text { Case Reports in } \\
\text { Gastroenterology }\end{array}$} & \multirow{2}{*}{\multicolumn{2}{|c|}{ Case Rep Gastroenterol 2013;7:327-331 }} \\
\hline & & \\
\hline & $\begin{array}{l}\text { DOI: 10.1159/000354802 } \\
\text { Published online: August 16, } 2013\end{array}$ & $\begin{array}{l}\text { (c) } 2013 \text { S. Karger AG, Basel } \\
\text { 1662-0631/13/0072-0327\$38.00/0 } \\
\text { www.karger.com/crg }\end{array}$ \\
\hline & \multicolumn{2}{|c|}{$\begin{array}{l}\text { This is an Open Access article licensed under the terms of the Creative Commons } \\
\text { Attribution-NonCommercial } 3.0 \text { Unported license (CC BY-NC) (www.karger.com/OA } \\
\text { license), applicable to the online version of the article only. Distribution permitted for non } \\
\text { commercial purposes only. }\end{array}$} \\
\hline
\end{tabular}
commercial purposes only.

\title{
Multiple Liver Lesions in an Immunosuppressed Patient: Is Infection Always the Answer?
}

\author{
Nihal M. Hamouda ${ }^{a}$ Rayna M. Grothe ${ }^{b}$ Samar H. Ibrahim ${ }^{b}$ \\ ${ }^{a}$ Department of Pediatrics and Adolescent Medicine and ${ }^{b}$ Division of Pediatric \\ Gastroenterology and Hepatology, Mayo Clinic, Rochester, Minn., USA
}

\section{Key Words}

Inflammatory bowel disease · Pylephlebitis · Liver abscess · Focal fatty liver disease

\begin{abstract}
Pylephlebitis with associated liver abscesses is a rare complication of Crohn's disease that usually presents in the setting of active disease and recent escalation of the immunosuppressive regimen. Here we describe a 17-year-old female patient with active Crohn's disease who presented with a clinical picture and hepatic radiographic findings concerning for pylephlebitis and associated liver abscesses but subsequently confirmed to be an atypical form of focal fatty liver infiltration. We describe the diagnostic difficulty this unique distribution of hepatic fatty infiltration can present and highlight the importance of the different imaging studies to differentiate between atypical focal fatty infiltration and pylephlebitis.
\end{abstract}

(C) 2013 S. Karger AG, Basel

\section{Introduction}

Pylephlebitis, or septic thrombophlebitis of the portal vein and/or its tributaries with associated liver abscesses, is a rare complication of Crohn's disease (CD) that often presents in the setting of poorly controlled disease and increased immunosuppressive therapy, secondary to suppuration in the region drained by the portal system [1]. We describe a patient with active $\mathrm{CD}$ who initially presented with a clinical picture and hepatic radiographic findings raising concern for pylephlebitis and associated liver abscesses but subsequently confirmed to be an atypical form of focal fatty liver disease.

Fatty infiltration of the liver can present with both diffuse and focal forms and is generally caused by increased levels of triglycerides in hepatocytes. While focal fatty infiltration is 
Hamouda et al.: Multiple Liver Lesions in an Immunosuppressed Patient: Is Infection Always the Answer?

not often considered in children, predisposing conditions in children and young adults are the same as for diffuse fatty infiltration and include diabetes, parenteral nutrition, cystic fibrosis, inherited metabolic diseases, chemotherapy, steroid use, malnutrition and obesity. Typically focal fatty infiltration of the liver does not display mass effect, and branches of the hepatic and portal veins traverse it without changing their course. Focal fatty infiltration of the liver can be transient in nature and may not be consistently seen on radiographic examinations. Focal fatty infiltrations are typically located adjacent to the falciform ligament, gallbladder fossa or porta hepatis. The characteristic location and lack of mass effect on imaging studies in children and young adults who have no evidence of liver disease support the diagnosis of focal fatty infiltration of the liver [2]. On the other hand, pylephlebitis and associated liver abscesses display a branching pattern on radiographic imaging. To the best of our knowledge, our patient is the first reported case of atypical distribution of focal fatty liver disease with poorly controlled inflammatory bowel disease (IBD).

\section{Case Report}

A 17-year-old female with long-standing fibrostenotic CD necessitating ileocecostomy presented with a 3-week history of worsening abdominal pain and increased stool frequency. Additionally, she reported increased fatigue and recent initiation of $17.5 \mathrm{mg}$ of methotrexate weekly for enteropathic arthritis. Physical examination was remarkable for mild abdominal tenderness to palpation. Medications at the time included $9 \mathrm{mg}$ of budesonide per day and adalimumab $40 \mathrm{mg}$ weekly in addition to methotrexate. Computed tomography (CT) enterography revealed active inflammation of the ileum as well as multiple small branching low-density areas in the inferior aspect of the right lobe of the liver and near the porta hepatis, suggestive of small fluid collections versus thrombosed peripheral veins (fig. 1). Due to concern for hepatic abscesses and CD exacerbation, she was subsequently admitted to the hospital for broad-spectrum intravenous antibiotic therapy. Laboratory workup revealed: hemoglobin $10.8 \mathrm{~g} / \mathrm{dl}$, leukocyte count $6.3 \times 10^{9} / \mathrm{l}$, platelets $459 \times 10^{9} / \mathrm{l}$, aspartate aminotransferase $18 \mathrm{U} / \mathrm{l}$ (normal 8-43), alanine aminotransferase $8 \mathrm{U} / \mathrm{l}$ (normal 7-45), alkaline phosphatase $102 \mathrm{U} / \mathrm{l}$ (normal 52-144), and total bilirubin $0.2 \mathrm{mg} / \mathrm{dl}$ (normal 0.1-1.0). C-reactive protein was elevated up to $13.7 \mathrm{mg} / \mathrm{l}$ and sedimentation rate was $24 \mathrm{~mm} / \mathrm{h}$. A coagulation panel was notable for an elevated D-dimer of $820 \mathrm{mg} / \mathrm{ml}$ and elevated fibrinogen of $472 \mathrm{mg} / \mathrm{dl}$. Further history and physical examination revealed no recent fever, right upper quadrant pain, tenderness or hepatomegaly. Review of the CT scan revealed patent portal, mesenteric and splenic veins.

To confirm our working diagnosis, a liver ultrasound with Doppler was performed and demonstrated several small, ill-defined, echogenic areas near the porta hepatis and in the inferior aspect of the right lobe of the liver, corresponding to the low attenuation area on the CT enterography, with sonographic appearance most consistent with focal fatty infiltration (fig. 2), and a patent portal vein. Magnetic resonance imaging of the abdomen with fat saturation confirmed that the hepatic lesions represented focal areas of fatty deposition. The patient's blood cultures did not grow any organisms, intravenous antibiotics were discontinued, methotrexate was optimized at $25 \mathrm{mg}$ per week, and the patient was discharged home on a taper of budesonide. 
Hamouda et al:: Multiple Liver Lesions in an Immunosuppressed Patient: Is Infection Always the Answer?

\section{Discussion}

Focal fatty liver infiltration is a much less common condition than the diffuse form. Typical locations for focal fatty liver deposition include the gallbladder fossa, the medial segment near the falciform ligament and at the porta hepatis [3]. Fatty liver disease is well recognized in patients with CD. In one study of 511 patients with IBD, about $40 \%$ of patients with CD had some degree of diffuse liver steatosis [4]. In another study of IBD patients receiving corticosteroids, $43 \%$ of patients with $\mathrm{CD}$ had focal fatty changes in the liver hilum [5]. It has to be noted that hepatic steatosis was not related to the site, duration or activity of disease. The atypical branching peripheral pattern of focal fatty deposition seen in our patient has not been described in the above study. Atypical patterns of hepatic fatty infiltration present significant diagnostic difficulty in differentiation from other pathological processes, especially liver abscesses, and often require additional radiological investigation.

Pylephlebitis, or septic thrombophlebitis of the portal vein, occurs secondary to suppuration in the region drained by the portal system or one of its tributaries. Inflammation, or interaction with the infectious agent and the endothelium, may precipitate the clotting cascade, which results in pylethrombosis [6]. Pylephlebitis is a rare complication of intraabdominal infections and is associated with significant morbidity and mortality if left untreated. Diverticulitis and appendicitis are the most common inciting infections, but there have been few reported cases of pylephlebitis attributed to IBD [6, 7]. Pylephlebitis typically occurs secondary to translocation of gut flora to the portal vein through the inflamed gut mucosa [7]. Thromboembolic compilations in patients with IBD are associated with active disease, previous abdominal surgery and sepsis [8]. Clinical manifestations of pylephlebitis are often nonspecific and most commonly include abdominal pain and fever. Less common presentations include right upper quadrant tenderness, hepatomegaly and, rarely, jaundice. The diagnosis is made with imaging studies demonstrating portal and/or mesenteric vein thrombosis, with associated liver abscesses along the terminal branch of the portal vein. Bacteremia is also present in a large percentage of patients [9]. Ultrasound with Doppler and CT scan can both demonstrate echogenic material within the portal vein suggestive of thrombus, but CT has the advantage of mesenteric and splenic vein thrombosis detection. Broad-spectrum polymicrobial antibiotic therapy is the mainstay of treatment and should be continued for a minimum of 6 weeks [1]. Anticoagulation has been suggested in conjunction with antibiotic therapy for pylephlebitis but remains controversial [1, 9].

In conclusion, focal fatty infiltration can occur in atypical locations in the liver and presents a diagnostic dilemma in patients with poorly controlled IBD and increased immunosuppressive therapy. The most important differential is pylephlebitis with associated hepatic abscesses. Patency of the portal vein and its tributaries, as in our case, can help exclude the condition. Liver ultrasound with Doppler is the noninvasive, cost-effective imaging study of choice to confirm the diagnosis and avoid undue use of prolonged antibiotics.

\section{Disclosure Statement}

The authors have no potential conflict of interest in regards to this paper. There was no funding. 


\section{Case Reports in
Gastroenterology}

\begin{tabular}{l|l}
\hline Case Rep Gastroenterol 2013;7:327-331 \\
\hline DOI: 10.1159/000354802 & $\begin{array}{l}\text { @ 2013 S. Karger AG, Basel } \\
\text { www.karger.com/crg }\end{array}$ \\
\hline
\end{tabular}

Hamouda et al.: Multiple Liver Lesions in an Immunosuppressed Patient: Is Infection Always the Answer?

\section{References}

1 Aguas M, Bastida G, Nos P, Beltran B, Grueso JL, Ponce J: Septic thrombophlebitis of the superior mesenteric vein and multiple liver abscesses in a patient with Crohn's disease at onset. BMC Gastroenterol 2007;12: 7-22.

-2 Kammen BF, Pacharn P, Thoeni RF, Lu Y, Qayyum A, Coakly F, Gooding CA, Brasch RC: Focal fatty infiltration of the liver: analysis of prevalence and CT findings in children and young adults. AJR Am J Roentgenol 2001;177:1035-1039.

3 Bhatnagar G, Sidhu HS, Vardhanabhuti V, Venkatanarasimha N, Cantin P, Dubbins P: The varied sonographic appearances of focal fatty liver disease: review and diagnostic algorithm. Clin Radiol 2012;67:372-379.

-4 Bargiggia S, Maconi G, Elli M, Molteni P, Ardizzone S, Parente F, Todaro I, Greco S, Manzionna G, Bianchi Porro G: Sonographic prevalence of liver steatosis and biliary tract stones in patients with inflammatory bowel disease: study of 511 subjects at a single center. J Clin Gastroenterol 2003;36:417-420.

-5 Dietrich CF, Schall H, Kirchner J, Seifert H, Hermann G, Caspary WF, Lembcke B: Sonographic detection of focal changes in the liver hilus in patients receiving corticosteroid therapy. Z Gastroenterol 1997;35: 1051-1057.

6 Baddley JW, Singh D, Correa P, Persich NJ: Crohn's disease presenting as septic thrombophlebitis of the portal vein (pylephlebitis): case report and review of the literature. Am J Gastroenterol 1999;94:847-849.

7 Tung JY, Johnson JL, Liacouras CA: Portal-mesenteric pylephlebitis with hepatic abscesses in a patient with Crohn's disease treated successfully with anticoagulation and antibiotics. J Pediatr Gastroenterol Nutr 1996;23:474-478.

-8 Talbot RW, Heppell J, Dozois RR, Beart RW: Vascular complications of inflammatory bowel disease. Mayo Clin Proc 1986;61:140-145.

-9 Plemmons RM, Dooley DP, Longfield RN: Septic thrombophlebitis of the portal vein: diagnosis and management in the modern era. Clin Infect Dis 1995;21:1114-1120.
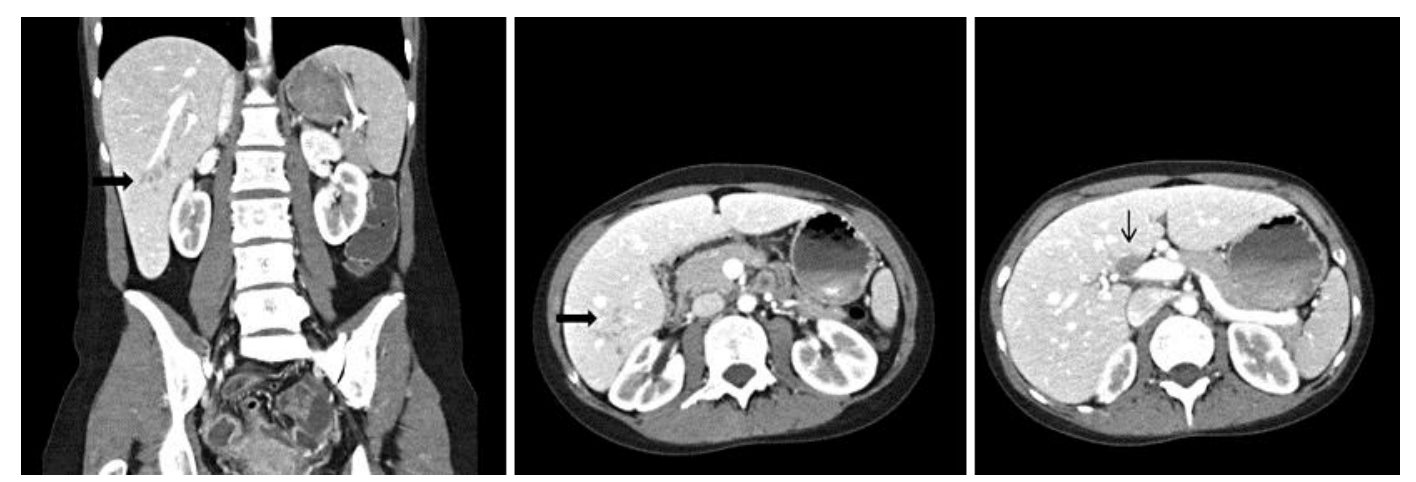

Fig. 1. CT enterography showing multiple small branching low-density areas in the inferior aspect of the right lobe (thick black arrows) and near the porta hepatis (thin black arrow). 
Hamouda et al.: Multiple Liver Lesions in an Immunosuppressed Patient: Is Infection Always the Answer?
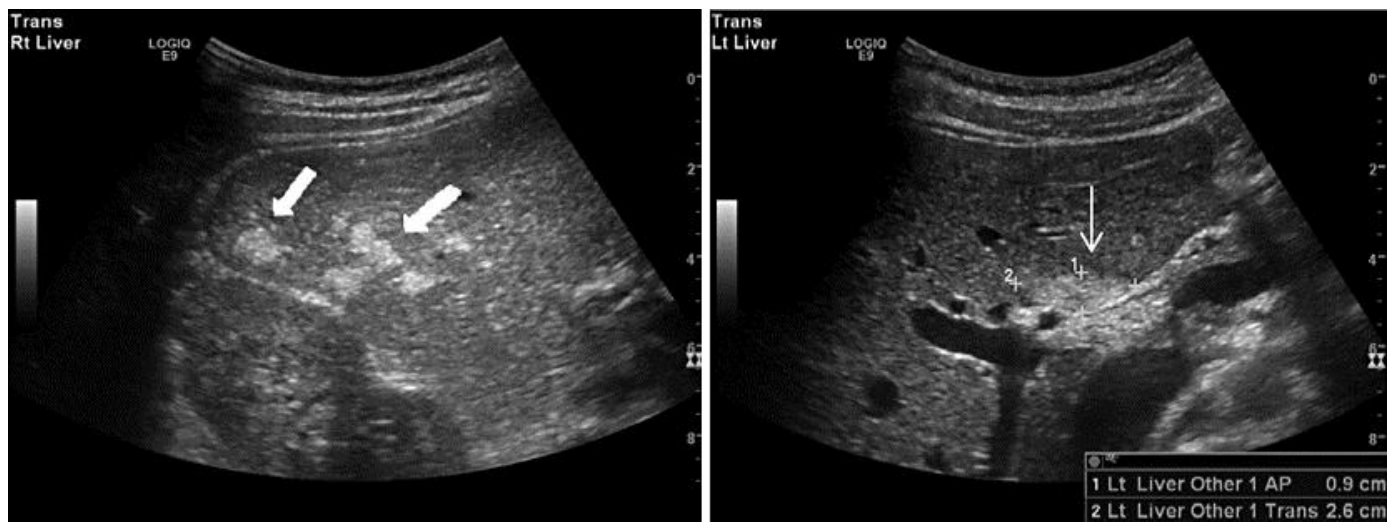

Fig. 2. Liver ultrasound showing small echogenic areas near the porta hepatis (thin white arrow) and the inferior aspect of the right lobe of the liver (thick white arrows), consistent with focal fatty infiltration. 